\title{
REPERCUSSÃO DA ESTIMULAÇÃO AUDITIVA RÍTMICA COM MÚSICA NO HUMOR DE PESSOAS COM DOENÇA DE PARKINSON
}

Cleysiane de Araujo Oliveira; Universidade Federal de Pernambuco; cleysiane.araujo@ufpe.br Ihana Thaís Guerra de Oliveira Gondim; Universidade Federal de Pernambuco; ihanafisio@hotmail.com Izaura Muniz Azevedo; Universidade Federal de Pernambuco; izauram73@hotmail.com Cilene Rejane Ramos Alves; Universidade Federal de Pernambuco; cilene.alves@ufpe.br Nadja Maria Jorge Asano; Universidade Federal de Pernambuco; nadja.asano@ufpe.br Jaqueline Severo dos Santos; Universidade Federal de Pernambuco; jaqueline.ssantos@ufpe.br Kássia Maria Clemente da Silva; Universidade Federal de Pernambuco; kassia.maria@ufpe.br Maria das Graças Wanderley de Sales Coriolano; Universidade Federal de Pernambuco; mariagracas.coriolano@ufpe.br

\section{RESUMO}

A depressão e o suicídio tem se tornado nas últimas décadas temáticas de relevância para a saúde pública. Dentre os fatores psicossociais envolvidos destaca-se o surgimento de doenças como a doença de Parkinson (DP). O tratamento farmacológico da sintomatologia depressiva na DP é realizado com uso de antidepressivos, no entanto a abordagem não farmacológica através da música pode ser utilizada como estímulo para o alívio dos sintomas não motores, tais como a depressão. O objetivo desse estudo é avaliar a repercussão da Estimulação Auditiva Rítmica (EAR) com música no estado de humor em pessoas com DP, com ênfase na sintomatologia depressiva. Esse é um estudo quase experimental (CAAE $n \div 29478620.7 .0000 .5208$ ) realizado para testar o uso de um aplicativo de smartphone com EAR com música sobre a sintomatologia depressiva na DP. Foi utilizado o inventário de depressão de Beck (BDI) antes e depois da intervenção. Com 21 itens, o BDI possibilita classificar a intensidade da sintomatologia depressiva. Os dados foram tabulados e a comparação pareada foi realizada através de teste $\mathrm{T}$, considerando $\mathrm{P}<0,05$. Resultados: Dez pacientes com DP leve a moderada, com média de idade de $64( \pm 10)$ anos, sendo 6 homens, sem uso de antidepressivos, concluíram a intervenção. A média do escore total do BDI antes da avaliação foi 9,4 $( \pm 7,9)$ e após a intervenção foi de 5,0 $( \pm 4,4)$, sendo esta diferença estatisticamente significativa $(\mathrm{P}=0,026)$. Os resultados obtidos até o momento na análise pareada revelam que a intervenção com EAR com música melhorou o estado de humor dos pacientes avaliados.

Palavras-chave: Doença de Parkinson; Depressão; Música; Estimulação Acústica.

Agradecimentos: a CNPq e a propesq UFPE pelo apoio financeiro. 